\title{
The Fear Circuit Revisited: Contributions of the Basal Amygdala Nuclei to Conditioned Fear
}

\author{
Taiju Amano, ${ }^{\star}$ Sevil Duvarci, ${ }^{\star}$ Daniela Popa, and Denis Paré \\ Center for Molecular and Behavioral Neuroscience, Rutgers State University, Newark, New Jersey 07102
}

The lateral nucleus (LA) is the input station of the amygdala for information about conditioned stimuli (CSs), whereas the medial sector of the central nucleus (CeM) is the output region that contributes most amygdala projections to brainstem fear effectors. However, there are no direct links between LA and CeM. As the main target of LA and with its strong projection to CeM, the basomedial amygdala (BM) constitutes a good candidate to bridge this gap. Consistent with this notion, it was reported that combined posttraining lesions of the basal nuclei [BM plus basolateral nucleus (BL)] abolish conditioned fear responses, whereas selective BL inactivation does not. Thus, we examined the relative contribution of BM and BL to conditioned fear using unit recordings and inactivation with muscimol microinfusions in rats. Approximately $30 \%$ of BM and BL neurons acquired robust responses to auditory CSs predicting footshocks. While most BL cells stopped firing at CS offset, BM responses typically outlasted the CS by $\geq 40 \mathrm{~s}$, paralleling the persistence of conditioned fear after the CS. This observation suggests that BM neurons are not passive relays of rapidly adapting LA inputs about the CS. Surprisingly, independent inactivation of either BM or BL with muscimol did not cause a reduction of conditioned freezing even though an extinction recall deficit was seen the next day. In contrast, combined BL-BM inactivation did. Overall, there results support the notion that the basal nuclei are involved in conditioned fear expression and extinction but that there is functional redundancy between them.

\section{Introduction}

The cellular substrates of classical fear conditioning continue to be the object of intense scrutiny because they are thought to contribute to human anxiety disorders (Davis, 1992; Phelps and LeDoux, 2005; Pape and Paré, 2010). Given the strategic position of the basomedial nucleus (BM) in the intraamygdaloid network, it is surprising that its potential role in fear conditioning has received little attention so far. Indeed, there are no direct connections between the lateral amygdala (LA), the main input station of the amygdala for thalamic and cortical information about conditioned stimuli (CSs) (LeDoux et al., 1990; Turner and Herkenham, 1991; Romanski et al., 1993), and the medial sector of the central nucleus (CeM) (Krettek and Price, 1978; Smith and Paré, 1994; Pitkänen et al., 1997), the region that contributes most amygdala projections to brainstem fear effectors (Hopkins and Holstege, 1978; Veening et al., 1984; Cassell et al., 1986; Gray and Magnuson, 1987; Rizvi et al., 1991). The lack of direct connections between the input and output stations of the amygdala for conditioned fear suggests that one or more target(s) of LA relay CS-related information to CeM. BM constitutes an obvious candidate for this role because it is the main target of LA (Krettek and

\footnotetext{
Received July 5, 2011; revised Aug. 12, 2011; accepted Sept. 5, 2011.

Author contributions: D. Paré designed research; T.A., S.D., and D. Popa performed research; T.A., S.D., and D. Paré analyzed data; D. Paré wrote the paper.

This work was supported by NIMH Grant R01 MH-083710. We thank Dr. T. Sigurdsson for his advice on data analysis and unit recording procedures.

*T.A. and S.D. contributed equally to this work.

Correspondence should be addressed to Denis Paré, Center for Molecular and Behavioral Neuroscience, Rutgers State University, 197 University Avenue, Newark, NJ 07102. E-mail: pare@andromeda.rutgers.edu.

DOI:10.1523/JNEUROSCI.3410-11.2011

Copyright $\odot 2011$ the authors $\quad 0270-6474 / 11 / 3115481-09 \$ 15.00 / 0$
}

Price, 1978; Smith and Paré, 1994; Pitkänen et al., 1997) and it projects to CeM (Paré et al., 1995; Pitkänen et al., 1997).

One factor explaining why potential BM contributions to learned fear have been neglected is that the first tests of its involvement in fear conditioning yielded negative results with pretraining lesions of the basal nuclei [BM plus basolateral nucleus (BL)] having little or no effect (Amorapanth et al., 2000; Goosens and Maren, 2001; Nader et al., 2001). However, new evidence suggests that a reexamination of its role is warranted. Indeed, a subsequent study, while confirming that combined pretraining $\mathrm{BL}-\mathrm{BM}$ lesions had no effect, revealed that postconditioning lesions led to the abolition of conditioned fear responses (AngladaFigueroa and Quirk, 2005). This pattern of results suggested that in an intact brain, the basal nuclei are at least required to relay potentiated CS-evoked LA responses to CeM but that, in their absence, LA can affect CeM via another route.

Because the study by Anglada-Figueroa and Quirk (2005) involved combined BL-BM lesions, it remained unclear whether $\mathrm{BM}, \mathrm{BL}$, or both were the critical lesion site(s) for the abolition of conditioned fear. However, it was later reported that selective posttraining inactivation of $\mathrm{BL}$ with muscimol does not interfere with conditioned fear responses (Herry et al., 2008). The contrasting results obtained in the latter two studies led us to hypothesize that BM, not BL, is the critical relay station between LA and CeM. Therefore, the present study was undertaken to investigate the relative contribution of $\mathrm{BM}$ and $\mathrm{BL}$ to the expression of conditioned fear responses using unit recordings and posttraining inactivation with muscimol.

\section{Materials and Methods}

Procedures were approved by the Institutional Animal Care and Use Committee of Rutgers University, in compliance with Guide for the Care 
and Use of Laboratory Animals (Department of Health and Human Services). Our subjects were adult male Sprague Dawley rats (Charles River Laboratories) maintained on a $12 \mathrm{~h}$ light/dark cycle. They were housed individually with ad libitum access to food and water. Before the experiments, they were habituated to the animal facility and handling for 1 week.

Unit recordings. Rats were anesthetized with isoflurane and administered atropine methyl nitrate $(0.05 \mathrm{mg} / \mathrm{kg}, \mathrm{i} . \mathrm{m}$. $)$ to reduce secretions and aid breathing. In aseptic conditions, rats were placed in a stereotaxic apparatus with nonpuncture ear bars. A local anesthetic (bupivacaine, subcutaneous) was injected in the region to be incised. The scalp was incised, small holes were drilled in the skull above the amygdala, and movable bundles of six to eight microwires ( $100-300 \mathrm{k} \Omega$ following gold plating) were stereotaxically aimed to BM or BL. The reference electrode was fixed to the skull overlying the cerebellum. At the end of the surgery, the wound was sutured and the rats were administered an analgesic with a long half-life (ketoprophen, $2 \mathrm{mg} / \mathrm{kg}$, s.c., daily for $3 \mathrm{~d}$ ) and $1 \mathrm{ml}$ of lactated Ringer's (subcutaneous). To minimize the risk of infection, a local antibiotic was applied directly on the wound (Neosporin paste). Rats were allowed at least 1 week to recover from the surgery.

We used conditioning chambers (Coulbourn Instruments) with metal grid floors and Plexiglas walls. Fear conditioning occurred in context A, the conditioning chamber described above. For extinction training and testing in context $\mathrm{B}$, the chamber was modified by introducing a black Plexiglas floor washed with peppermint soap. On day 0 , rats were habituated to contexts A and B for 15 min each in a counterbalanced manner. On day 1 , the electrodes were lowered, and $1-2 \mathrm{~h}$ later, the rats received a tone [conditioned stimulus (CS); $4 \mathrm{kHz}, 80 \mathrm{~dB}$ ] habituation session that was followed by a fear conditioning session in which the rats received five presentations of the CS, each coterminating with a footshock unconditioned stimulus (US) $(0.5 \mathrm{~mA}, 1 \mathrm{~s})$. On day 2, rats underwent an extinction training session consisting of 20 presentations of the CS alone in context B (CS 1-5, fear recall; CS 16-20, extinction). On day 3, extinction recall was tested with 10 additional presentations of the CS alone in context B. Behavior was recorded by a video camera and scored off-line. Time spent freezing (immobility, with the exception of breathing) was measured. At the end of behavioral experiments, electrolytic lesions were performed to mark the recording sites.

Unit activity was sampled at $25 \mathrm{kHz}$ and stored on a hard drive. Data were analyzed off-line with custom software written in MATLAB. Spike sorting was performed off-line using a clustering algorithm based on principal component analysis and $K$-means. Because the spike shapes of most units changed from day to day, it is assumed that different cells were recorded on each of the three daily recording sessions.

To analyze CS-evoked changes in activity, the firing rate of each unit was computed in $5 \mathrm{~s}$ bins, from $20 \mathrm{~s}$ before to $100 \mathrm{~s}$ after CS onset. Separate averages of the five CSs of the tone habituation phase, the last three CSs of fear conditioning, first five and last five CSs of the extinction training session and the first and last five CSs of the extinction testing session were calculated. The data were then $z$ scored by first subtracting the pre-CS average firing rate from the data and then dividing by the SD of the pre-CS period.

For cell-by-cell analyses, CS and baseline periods were compared for each cell and the cells that showed $\geq 1$ bin where their firing rate differed by greater than or equal to $\pm 2.5 z$ of baseline were considered to have a significant CS responsiveness.

Reversible inactivation with muscimol. Using the same surgical techniques as above, rats were bilaterally implanted with stainless-steel guide cannulas (26 gauge; Plastics One) aimed to BL, BM, or both. Dental cement was then used to fix the cannulas to the skull screws. A 33 gauge dummy cannula was inserted to prevent clogging.

Diffusion of fluorescent muscimol (Bodipy TMR-X-conjugated muscimol; Invitrogen) from the infusion site was assessed in 18 rats. These rats received the same volume $(0.3 \mu \mathrm{l} /$ hemisphere $)$ and concentration $[0.5 \mathrm{~mm}$ dissolved in artificial CSF (aCSF)] of muscimol as in the behavioral experiments. Ten minutes after the end of the infusion, they were perfused-fixed, and the extent of diffusion was assessed on coronal sections.

The fear conditioning protocol was conducted in the same conditioning chambers and contexts as used for the unit recording experiments.
On day 0 , rats were habituated to the contexts for 15 min each in a counterbalanced manner. On day 1 , after tone habituation $(4 \mathrm{kHz}, 80$ $\mathrm{dB})$, rats were fear conditioned with four pairings of the CS and US (0.5 $\mathrm{mA}, 1 \mathrm{~s})$. The CS and US coterminated. On day 2, the rats received intracerebral infusions of either muscimol or vehicle. Internal infusion cannulas were attached to $1.0 \mu \mathrm{l}$ microsyringes via polyethylene tubing filled with the solution to be infused $(0.1 \mu \mathrm{l} / \mathrm{min})$. After the infusions, the cannulas were left in place for 1 additional minute. Ten minutes after the conclusion of the muscimol infusions, the rats then received 20 presentations of the CS alone. On day 3, in the same animals drug-free, an extinction recall test was conducted (10 presentations of the CS alone).

Depending on the experiments, rats received bilateral vehicle or muscimol infusions in (1) BL, (2) BM, or (3) BL plus BM. In the latter experiment, rats received twice the quantity of muscimol as rats in Experiments 1 and 2, potentially resulting in significant muscimol diffusion to CeM. Thus, we performed control experiments in which rats received bilateral infusions in BM plus a site dorsal to CeM. The location of this site was chosen so that it would be equidistant from CeM as the $\mathrm{BL}$ infusion site of Experiment 3.

Histology. At the conclusion of the experiments, the rats were given an overdose of pentobarbital $(80 \mathrm{mg} / \mathrm{kg})$, perfused with $100 \mathrm{ml}$ of ice-cold PBS $(0.1 \mathrm{M}), \mathrm{pH} 7.4$, followed by $100 \mathrm{ml}$ of a fixative containing paraformaldehyde $(4 \%)$ in $0.1 \mathrm{~m}$ phosphate buffer, $\mathrm{pH}$ 7.4. The brain was then removed from the skull, postfixed overnight in the same fixative, and sectioned at $100 \mu \mathrm{m}$ on a vibrating microtome. Results (below) include only units recorded within histologically confirmed BM and BL locations. For the infusion experiments, we considered only rats with correct bilateral cannula placements.

\section{Results}

\section{CS-evoked activity in the basal nuclei during fear conditioning}

It was reported that $\mathrm{BL}$ inactivation with muscimol does not block fear expression (Herry et al., 2008), whereas combined BL-BM posttraining lesions do (Anglada-Figueroa and Quirk, 2005). A possible explanation for this apparent discrepancy is the differential involvement of BM in the two studies. According to this view, during CS presentations, LA cells would recruit BM neurons that project to CeM, thereby contributing to drive conditioned fear. If this were true, we would expect auditory fear conditioning to alter the CS responsiveness of BM cells. However, the behavior of BM cells during fear conditioning has not been examined so far (Pape and Paré, 2010). Our first experiment therefore aimed to test this prediction. For comparison, we also studied the activity of BL cells.

Histological controls revealed that microwire bundles reached $\mathrm{BM}$ in 11 rats (Fig. $1 \mathrm{~A}$ ). Off-line clustering of unit activity yielded a sample of 68 BM cells $(31,22$, and 15 on days $1-3$, respectively). Because the spike shapes of most units changed from day to day, below we assumed that different cells were recorded on different days.

Figure $1 B$ illustrates the time rats spent freezing during habituation, fear conditioning, as well as during the extinction training and testing sessions. The data are color-coded like the corresponding unit activity of Figure 1, $C$ and $D$. Expectedly, fear conditioning caused an increase in freezing levels (CS 3-5, 78.8 \pm $6.3 \%$ ) compared with habituation (CS $1-5,10.9 \pm 6.5 \%$; paired $t$ test, $p<0.0001$ ). The next day, in a different context, the first few CSs still elicited large increases in freezing (CS 1-5, $80.5 \pm 5.4 \%$ ) that gradually diminished with additional CS presentations (CS $16-20,13.4 \pm 8.1 \%$; paired $t$ test, $p<0.0001)$. Note that, during the recall test in context $\mathrm{B}$, rats showed little or no freezing (preCS, $16.4 \pm 8.7 \%$ ) until the CS was presented (Fig. $1 B$ ), indicating that conditioning occurred and that it was to the tone. On day 3 , the first few CSs elicited freezing (CS 1-5, $46.5 \pm 8.7 \%$ ) that again 

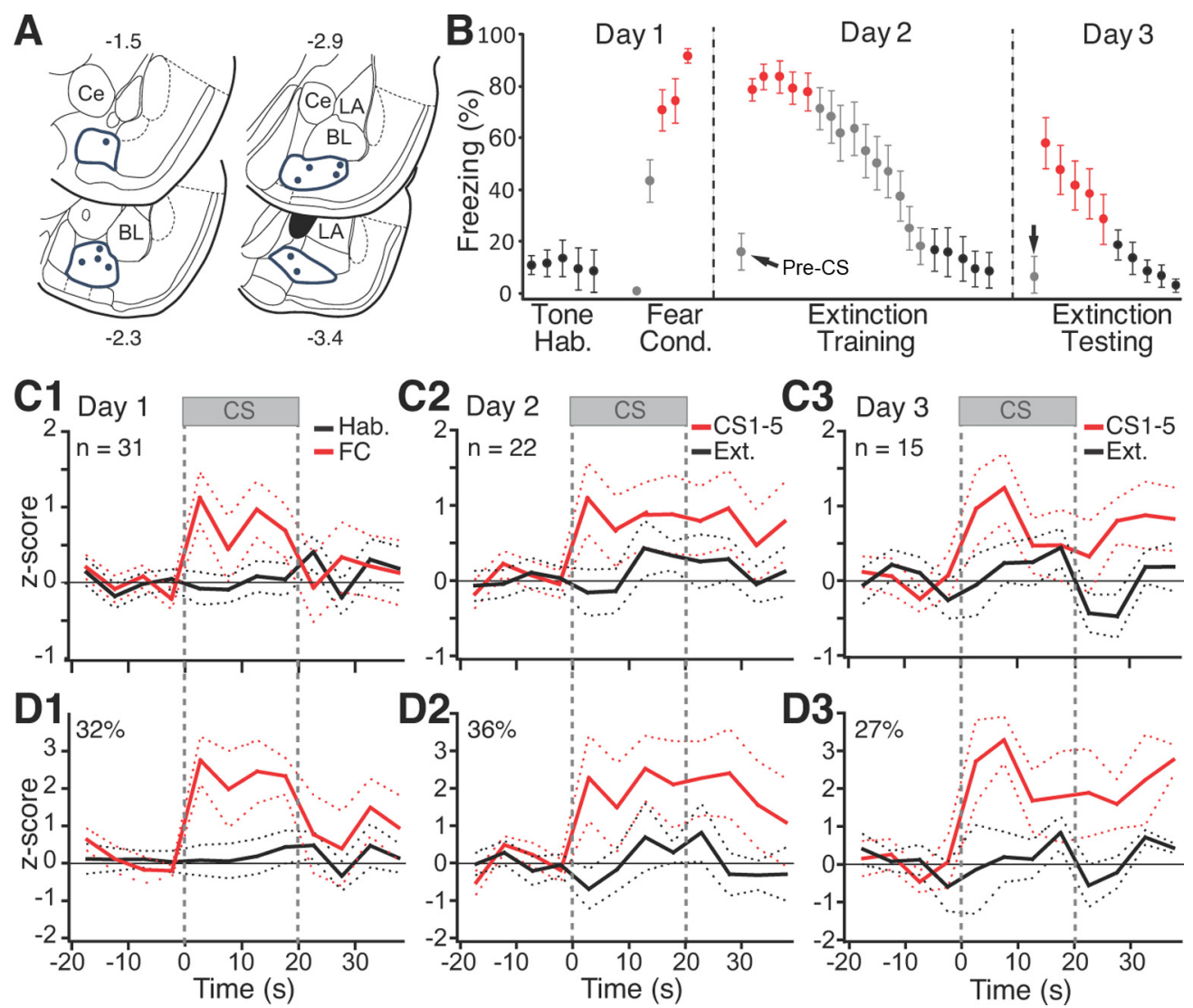

Figure 1. CS responsiveness of BM neurons. A, Locations of microwire bundles. B, Behavior of rats used for BM recordings. Hab., Habituation; Cond., conditioning. Error bars indicate SEM. C1-C3, Average CS responses of all cells recorded on days $1-3$, respectively. D1-D3, Average restricted to cells with significant increases in firing rate during $\geq 1$ bin of the CS (threshold, $\pm 2.5 z$ ).
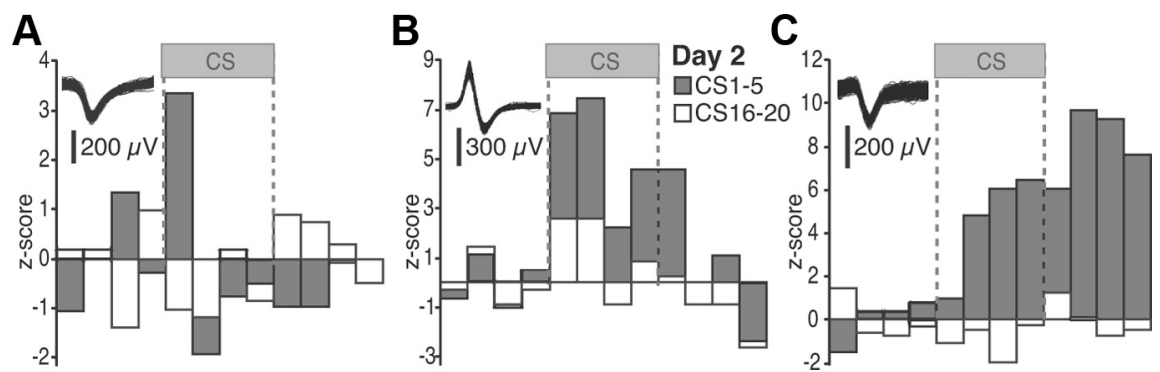

Figure 2. Representative examples of individual BM units. $A-C$ illustrate three different cells. The insets show action potential waveforms for each cell. Firing rates are $z$ scored to the baseline (pre-CS) period ( $5 \mathrm{~s}$ bins). The gray and white bars represent the average of the first and last five CSs of the recall test, respectively.

extinguished with additional presentations of the CS (CS 6-10, $2.1 \pm 0.8 \%)$.

To study CS-evoked activity, we calculated the firing rate of each unit in $5 \mathrm{~s}$ bins, from $20 \mathrm{~s}$ before to $100 \mathrm{~s}$ after CS onset. We obtained separate averages of the five CSs of the habituation phase, the last three CSs of training, as well as the first five and last five CSs of the extinction training and testing sessions, as indicated by the color-coded symbols of Figure $1 B$. We then $z$ scored the data. During habituation, the CS did not alter BM population activity (Fig. 1C1, black). However, by the end of fear conditioning (Fig. 1C1, red), the CS elicited significant firing rate increases (paired $t$ test, $p=0.0032$ ). The next day, BM neurons still displayed large CS-evoked responses (first five CSs, paired $t$ test, $p=0.015$; Fig. 1C2, red) that persisted long after CS offset (60 \pm $11.5 \mathrm{~s}$; range, $40-80 \mathrm{~s})$. Further CS presentations led to a marked reduction in CS responses (Fig. 1C2, black), paralleling fear extinction. During extinction testing, with spontaneous fear recovery, CS-evoked responses returned (Fig. 1C3, red) but diminished again with additional CS presentations (Fig. 1C3, black).

The results of cell-by-cell analyses (Fig. $1 D$ ) paralleled this average population behavior. To test this, we compared the CS and baseline periods and looked for cells that showed at least one $5 \mathrm{~s}$ bin during the CS in which their firing rate differed by more than $\pm 2.5 z$ of the baseline period (significance threshold of $p \leq 0.05$ ). At the end of conditioning (Fig. 1D1, red) and beginning of extinction training (Fig. 1D2, red) or testing (Fig. 1D3, red), 32, 36, and 27\% of cells, respectively, exhibited significant CS-evoked firing rate increases compared with none during habituation (Fig. 1D1, black). These changes in the incidence of CS responsive cells from low to high fear states were significant (Fisher's exact test, $p<0.0005$ ).

Representative examples of individual $\mathrm{BM}$ units during the first recall (day 2) test are provided in Figure $2 A-C$. In a few cells (9\%), the CS elicited rapidly adapting responses (Fig. 2A). Equally infrequent were cells exhibiting sustained responses throughout the CS but with little persistent activity at CS offset (Fig. $2 \mathrm{~B} ; 9 \%$ ). The incidence of cells exhibiting sustained responses during the CS plus persistent activity after CS offset (Fig. $2 C)$ was higher $(18 \%)$. Of the BM cells with significant CS re- 
A
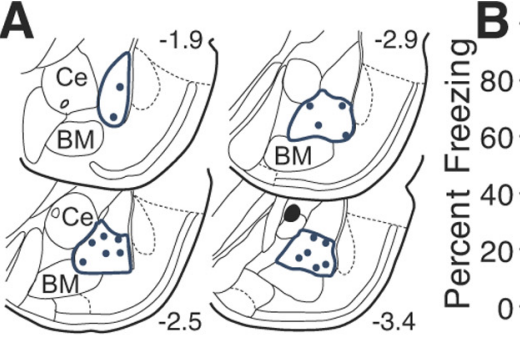
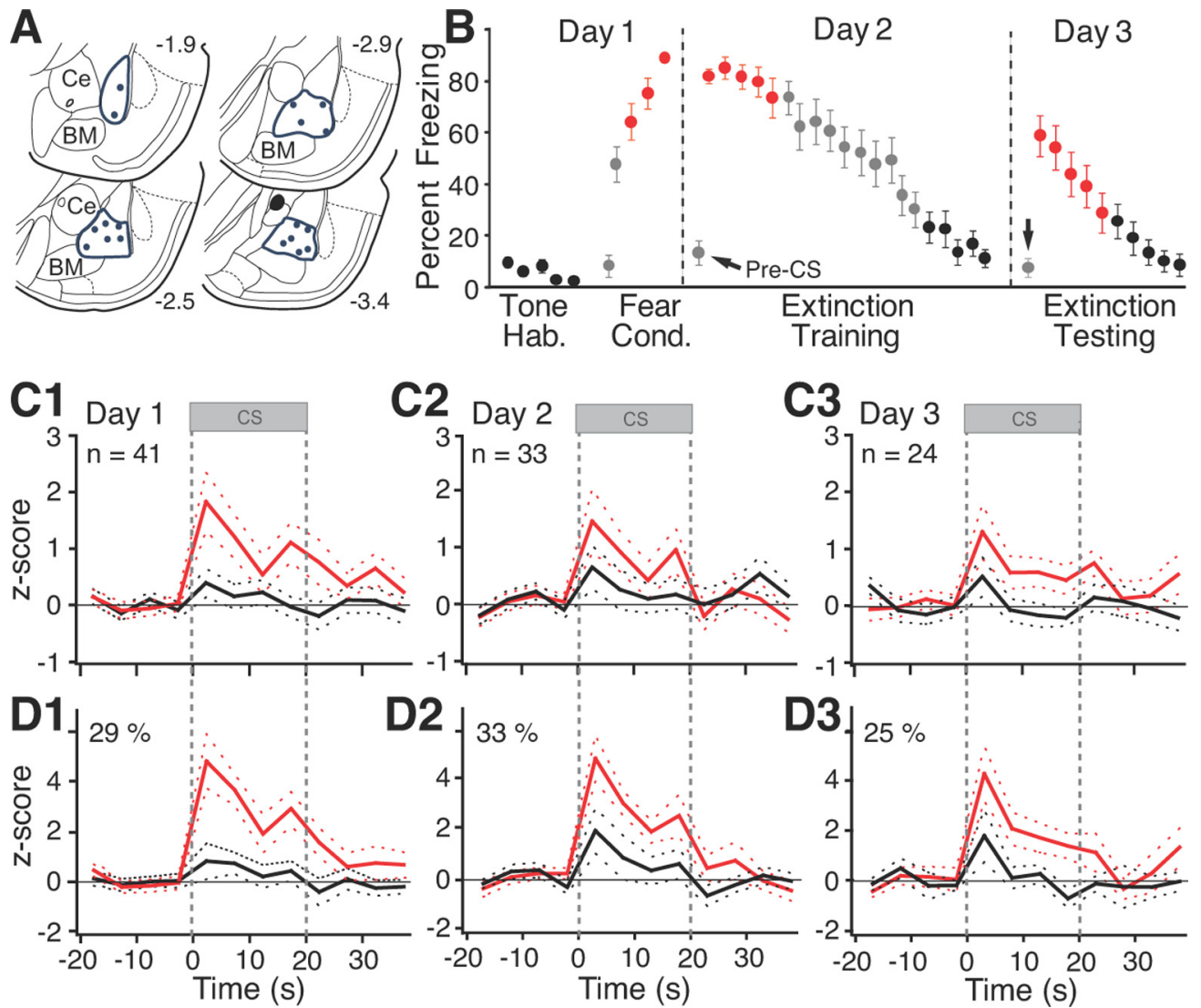

C2 Day 2

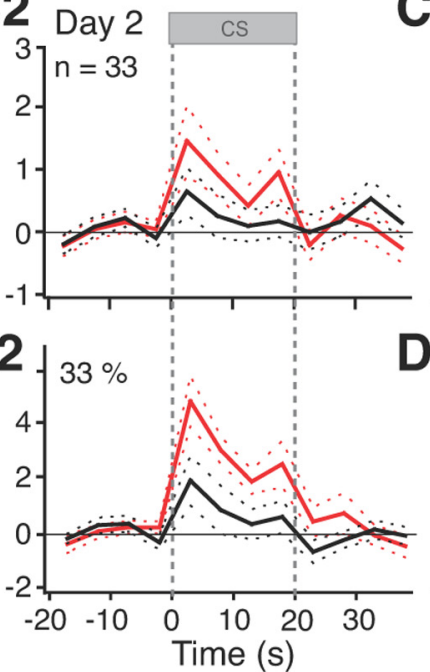

C3

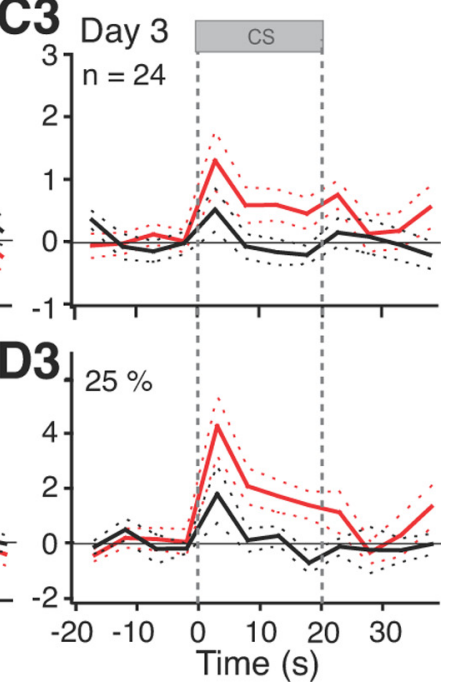

Figure 3. CS responsiveness of BL neurons. A, Locations of microwire bundles. B, Behavior of rats used for BL recordings. Error bars indicate SEM. C1-C3, Average CS responses of all cells recorded on days $1-3$, respectively. D1-D3, Average restricted to cells with significant increases in firing rate during $\geq 1$ bin of the CS (threshold, $\pm 2.5 z$ ).

sponses at the beginning of the recall test, most $(75 \%)$ became CS unresponsive by the end of extinction training (day 2). Although $25 \%$ of cells maintained their CS responsiveness despite extinction, no initially unresponsive BM neurons acquired CS responses as a result of extinction training (day 2). Using the $2.5 z$ threshold to assess significance, no units with inhibitory CS responses were detected.

The pattern of CS-evoked activity displayed by BL neurons was similar to that of BM cells, but with some interesting differences. Histological controls revealed that microwire bundles reached BL in 18 rats (Fig. 3A). Off-line clustering of unit activity recorded in these rats yielded a sample of 98 BL cells (41, 33 , and 24 on days $1-3$, respectively). Figure $3 B$ illustrates the percentage time these rats spent freezing during the behavior protocol. The data are color-coded like the corresponding unit activity of Figure 3, C1-C3 and D1-D3. The behavior of these rats was undistinguishable from that of rats used for the BM recordings ( $t$ tests on various phases of behavioral protocol, values of $p$ ranging between 0.26 and 0.88 ).

Figure $3 C$ shows the average behavior of all recorded BL neurons. As with $\mathrm{BM}$ cells, BL neurons were not $\mathrm{CS}$ responsive during habituation (Fig. 3C1, black) but showed robust tone responses by the end of fear conditioning (Fig. 3C1, red; paired $t$ test, $p=0.014$ ). At the onset of the extinction training session on day 2, the CS still elicited significant firing rate increases (Fig.

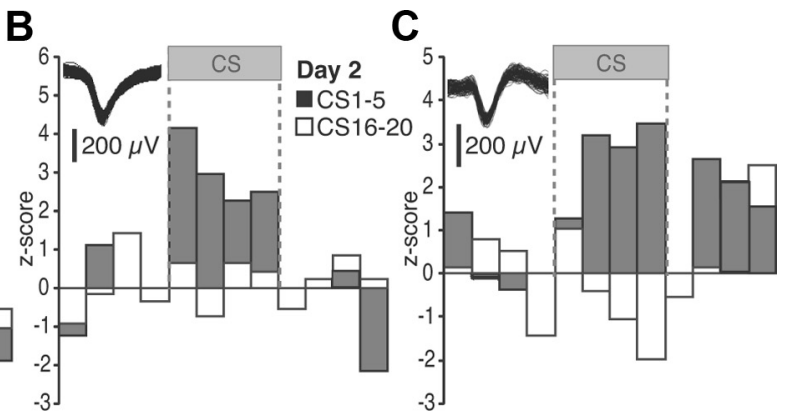

Figure 4. Representative examples of individual BL units. $A-C$ illustrate three different cells. The insets show action potential waveforms for each cell. Firing rates are $z$ scored to the baseline (pre- $C S$ ) period ( $5 \mathrm{~s}$ bins). The gray and white bars represent the average of the first and last five $C S$ s of the recall test, respectively.

$3 C 2$, red; paired $t$ test, $p=0.04$ ). However, in contrast with BM units, BL responses generally did not outlast the CS. Further CS presentations led to a marked reduction in $\mathrm{BL}$ tone responses (Fig. 3C2, black; paired $t$ test, $p=0.25$ ), paralleling fear extinction. At the start of the extinction testing session the next day, CS-evoked activity returned (Fig. 3C3, red; paired $t$ test, $p=$ $0.044)$, paralleling spontaneous fear recovery. These responses largely disappeared with additional CS presentations (Fig. 3C3, black; paired $t$ test, $p=0.91$ ).

The CS responses of individual BL neurons were consistent with the average behavior described above. Using the same criterion as for BM cells, cell-by-cell analyses revealed that during habituation, at the end of fear conditioning, and at the beginning of the extinction training and testing sessions, 2.4, 29, 33, and 
$25 \%$ of the cells, respectively, displayed significant CS responses. The higher proportion of tone responsive cells in high compared with low fear states was statistically significant (Fisher's exact test, $p<0.0005$ ). Representative examples of individual BL units during the first recall test (day 2) are provided in Figure 4. Although the range of CS response profiles seen in BL cells (Fig. $4 A-C$ ) was similar to that observed among BM neurons (Fig. $2 A-C$ ), their relative incidence differed. In particular, whereas cells with sustained activity after CS offset prevailed in BM, they were rarely seen in $\mathrm{BL}$ ( $3 \%$ or 1 of 33 ; Fig. $4 C$ ). The differing incidence of cells with sustained post-CS activity in BL and BM during the two recall tests was statistically significant (Fisher's exact test, $p<0.031$ ).

Of the BL cells with significant CS responses at the beginning of the recall test, most (73\%) became CS unresponsive by the end of extinction training (day 2). Although $27 \%$ of them maintained their CS responsiveness despite extinction, no initially unresponsive BL neurons acquired CS responses as a result of extinction training (day 2). Using the $2.5 z$ threshold to assess significance as for BM cells, no BL units with inhibitory CS responses were detected. However, inspection of individual peri-CS histograms such as the cases shown in Figure 4, $A$ and $C$, suggested that inhibitory CS responses did develop as a result of extinction training. This led us to suspect that the approach we used to assess significance (at least one bin $\geq 2.5 z$ ) was biased toward the detection of cells with large transient responses and missed lower amplitude but sustained responses. To test this idea, we averaged all CS bins and computed frequency distributions of $z$ scores at all phases of the experiment (Fig. 5A1-A3).

As shown in Figure 5, $A 2$ and $A 3$, in which the red and black curves denote data respectively obtained with the first five and last five CSs on day 2 (Fig. 5A2) and day 3 (Fig. 5A3), this approach confirmed that extinction training caused a negative shift in the distribution of $z$ scores to the left of zero (Fig. 5A2,A3, left arrows). Yet, even using a very liberal $z$ score threshold of -1.64 to assess significance in individual cells ( 0.05 significance level in a one-tailed test), none of these inhibitory responses reached significance. This approach also revealed that some BL cells had negative CS responses during the first recall test (CSs $1-5$ on day 2 ), and two of these exceeded $-1.64 z$ (Fig. $5 C$ ). Further examination of the data revealed that the $\mathrm{BL}$ cells with inhibitory CS responses during the recall test exhibited positive $z$ scores by the end of extinction training. In other words, their CS responses were transformed from an inhibition to an excitation. Hereafter, these cells will be termed "extinction cells." Conversely, many of the BL cells with positive responses during the recall test (hereafter termed "fear neurons") exhibited inhibitory CS responses (negative $z$ scores) by the end of extinction training.

To illustrate this point, we computed, cell by cell, the difference between the $z$ scores of the CS responses at early versus late stages of extinction, combining the data obtained on days 2 and 3 (Fig. $5 B$ ). Here, positive values on the $x$-axis indicate cells that were excited at the beginning of extinction and less so (or even inhibited) at the end of extinction. Conversely, negative values on the $x$-axis indicate cells that were inhibited (or poorly responsive) at the beginning of extinction and less inhibited (or excited) at the end of extinction. The circles indicate individual cells. The red circles indicate fear cells that had significant positive responses to CS $1-5$. The filled and empty red circles indicate cells with $z$ scores that did versus did not shift polarity in response to CS 16-20. Among the latter, cells that maintained significant positive CS responses at the end of extinction, hereafter termed extinctionresistant cells, are indicated by arrowheads and dashed lines. The
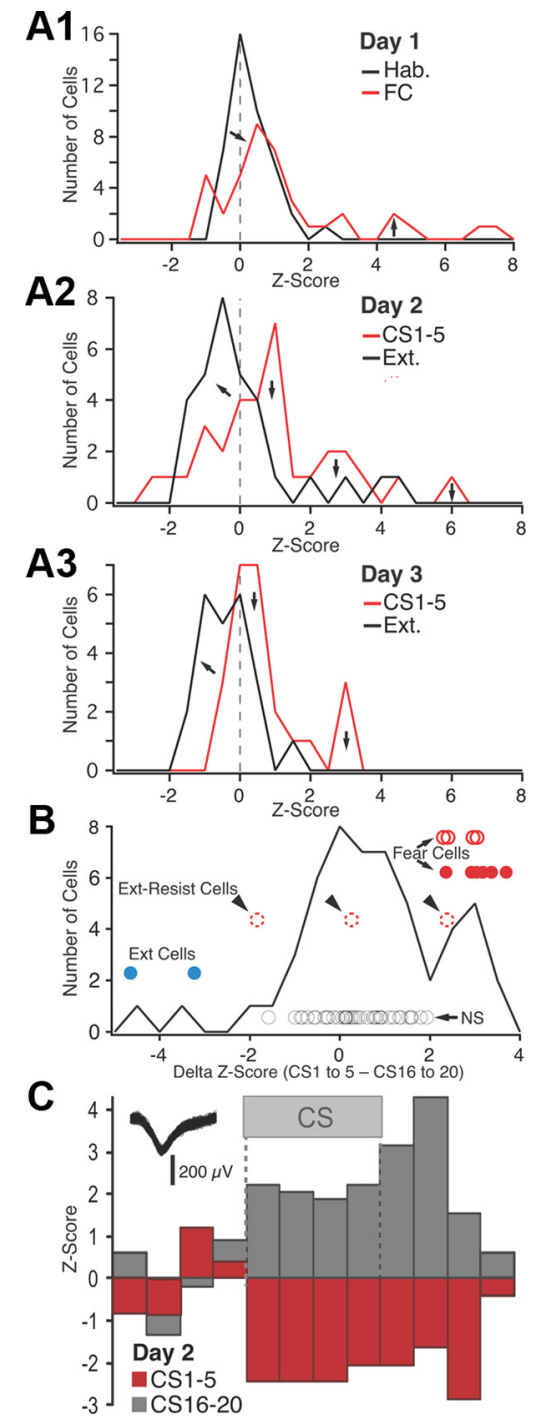

Figure 5. Frequency distribution of Z-scored CS responsiveness in BL neurons. $A$, Frequency distributions of $z$-scored $C S$ responses (based on average of the four $5 \mathrm{~s}$ CS bins) on day 1 (A1), day $2(\boldsymbol{A 2})$, and day $3(\boldsymbol{A} 3)$. See legend at top right corner of each panel for explanation of color coding. FC, Fear conditioning; Ext., extinction. $B$, Frequency distribution of the difference in $Z$ scores between the first five and last five CSs of extinction training (data obtained on days 2 and 3 are combined). The circles represent individual cells (see text). Their position with respect to the $y$-axis has no meaning; different cell subtypes are offset to various degrees for clarity. Blue, Extinction cells with significant negative $z$ scores in response to CS 1-5 and positive $z$ scores in response to the last five CSs. Red circles, Fear cells (filled red circles, fear cells that were inhibited by the end of extinction training; empty red circles with solid line, fear cells that became unresponsive by the end of extinction training; empty red circles with dashed lines, fear cells that maintained a significant positive response to the last 5 (Ss). Black circles, Cells with nonsignificant CS responses at all time points. $\boldsymbol{C}$, Example of an extinction cell.

blue circles indicate extinction cells, here liberally defined as cells that had negative $z$ scores in response to the first five CSs and positive $z$ scores in response to CS 16-20, at least one of which exceeded $\pm 1.64 z$ score. Finally, the black circles mark cells with no significant responses at all time points.

Overall, this analysis revealed that during extinction training, CS responses are transformed from a significant excitation to a significant inhibition in $46 \%$ of BL fear cells (Fig. $5 B$, solid red circles), $31 \%$ became unresponsive (Fig. $5 B$, empty red circles with continuous lines), and the rest (23\%; "extinction-resistant cells") continued to display significant positive CS responses (Fig. $5 B$, empty red circles with dashed lines). Even with the liberal 

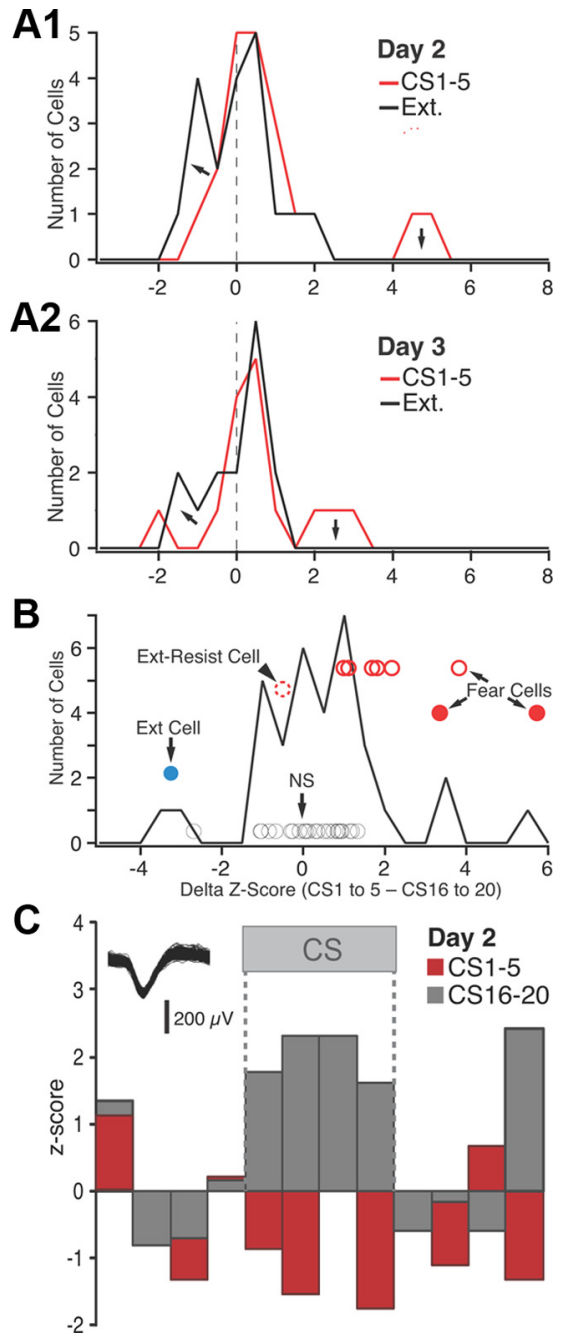

Figure 6. Frequency distribution of $z$-scored CS responsiveness in BM neurons. A, Frequency distributions of $z$-scored $C S$ responses (based on average of the four $5 \mathrm{~s} C \mathrm{~S}$ bins) on day 2 (A $\mathbf{T}$ ) and day 3 (A2). See legend at top right corner of each panel for explanation of color coding. $\boldsymbol{B}$, Frequency distribution of the difference in $z$ scores between the first five and last five CSs of extinction training (data obtained on days 2 and 3 are combined). The circles represent individual cells that reached significance (see text). Their position with respect to the $y$-axis has no meaning; different cell subtypes are offset to various degrees for clarity. Blue, Extinction cell with significant negative $z$ scores in response to $C S 1-5$ and positive $z$ scores in response to $C S$ $16-20$. Red circles, Fear cells (filled red circles, fear cells that were inhibited by the end of extinction training; empty red circles with solid line, fear cells that became unresponsive by the end of extinction training; empty red circles with dashed lines, fear cells that maintained a significant positive response to $C S 16-20)$. Black circles, Cells with nonsignificant $C S$ responses at all time points. $C$, Example of extinction cell.

definition used here, BL extinction cells were rare (2 of 57 or 3.5\%). See example in Figure 5C.

Qualitatively identical results were obtained in BM neurons. Extinction training caused a negative shift in the distribution of $z$ scores to the left of zero, on days 2 (Fig. 6A1) and 3 (Fig. 6A2). Yet, using a $z$ score threshold of -1.64 to assess significance in individual cells, none of these inhibitory responses reached significance. Some cells had negative CS responses during the first recall test (CS $1-5$ of extinction training), and one of these exceeded $-1.64 z$ (Fig. 6C).

Computing the difference between the $z$ score of CS responses of BM neurons at early versus late stages of extinction (Fig. 6B) revealed similar response variability as in $\mathrm{BL}$. That is, during extinction training, the CS responses of $22 \%$ BM fear cells was transformed from a significant excitation to a significant inhibition (Fig. 6B, solid red circles), 67\% became unresponsive (empty red circles with solid line), and $8 \%$ maintained a significant positive CS response (i.e., extinction-resistant cell, arrowhead). Similar to BL, only $3 \%$ of BM neurons (or 1 of 37) could be classified as an extinction cell (Fig. 6B, blue circle). See example in Figure 6C.

\section{Reversible inactivation of the basal nuclei during the expression of conditioned fear responses}

Overall, the above suggests that, as a result of fear conditioning, a substantial proportion of neurons in the basal nuclei acquire excitatory responses to the CS. Since previous tracing (Paré et al., 1995; Pitkänen et al., 1997) and electrophysiological (Royer et al., 1999) studies indicate that the basal nuclei send strong glutamatergic projections to $\mathrm{CeM}$, these findings suggest that CSresponsive neurons in the basal nuclei may contribute to the generation of conditioned fear responses. To further test this idea, we compared the effects of bilateral vehicle versus muscimol infusions in BL or BM, 10 min before testing fear recall.

\section{Control experiments on muscimol diffusion}

Since it was critical that we obtain very localized inactivations, we first performed a series of control experiments that assessed the extent of muscimol diffusion from the infusion site. We selected the same muscimol concentration and volume as used in the study by Wilensky et al. (2006) because, to our knowledge, it constitutes the lowest quantity of muscimol ever used to inactivate an amygdala nucleus in the fear conditioning literature.

Our control rats received unilateral infusions of fluorescent muscimol in $\mathrm{BL}(n=9$; Fig. $7 A)$ or $\mathrm{BM}(n=9$; Fig. $7 B)$. These rats received $0.3 \mu \mathrm{l}$ of a solution containing $0.5 \mathrm{~mm}$ fluorescent muscimol $(0.1 \mu \mathrm{l} / \mathrm{min})$ dissolved in aCSF, as we used for the behavioral experiments described below. Ten minutes after the infusion, the animals were perfused-fixed, their brains were extracted, and the extent of muscimol diffusion was examined. As shown in the representative example of Figure 7, $A$ and $B$, with these parameters, the infusion site was confined within the border of the targeted nucleus.

Impact of BL or BM inactivation

We first describe the behavior of rats that received bilateral vehicle $(n=10)$ or muscimol $(n=11)$ infusions in BL, 10 min before testing fear recall (Fig. $8 \mathrm{~A}$, arrow). In this and the following figure panels, the empty and filled bars are used for the vehicle and muscimol groups, respectively. The two groups exhibited similar levels of freezing by the end of the fear conditioning session ( $t$ test, $p=0.92$ ). The next day, muscimol infusions in BL performed 10 min before testing recall did not reduce fear expression $(t$ test, $p=$ 0.73 ), as previously reported (Herry et al., 2008).

Next, we tested whether BM activity was required during fear expression (vehicle, 13 rats; muscimol, 15 rats). However, muscimol infusions in BM (Fig. $8 B$ ) also failed to block freezing during fear recall ( $t$ test, $p=0.1$ ). It could be argued that the reason why $\mathrm{BL}$ or $\mathrm{BM}$ muscimol infusions failed to reduce conditioned fear is that we infused insufficient quantities of muscimol. However, this seems unlikely because, the next day, in the same animals drug-free, a between-session extinction deficit was seen relative to the animals infused with vehicle (vehicle vs muscimol, CS 1-2, $t$ tests: BL, $p=0.003$; BM, $p=0.002$ ).

Impact of combined BL-BM inactivations

Reasoning that functional redundancy between $\mathrm{BL}$ and $\mathrm{BM}$ might explain why independent inactivation of these two nuclei 

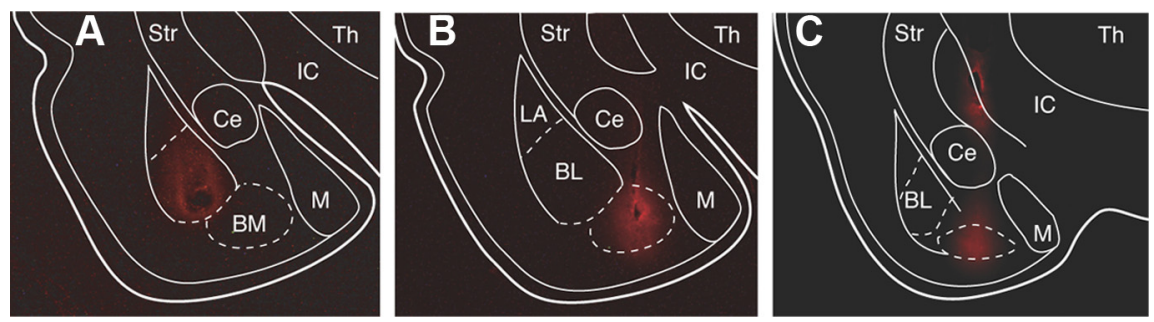

Figure 7. Extent of muscimol diffusion from the infusion site. At each infusion site, the rats received $0.3 \mu l$ of $0.5 \mathrm{~mm}$ fluorescent muscimol dissolved in aCSF, as in the behavioral experiments. $\boldsymbol{A}-\boldsymbol{C}$, Muscimol diffusion 10 min after infusion in $B L(\boldsymbol{A}), \operatorname{BM}(\boldsymbol{B})$, or in BM plus dorsal to $\mathrm{Ce}(\boldsymbol{C})$. Ce, Central nucleus of the amygdala; IC, internal capsule; M, medial nucleus of the amygdala; Str, striatum; Th, thalamus.

did not reduce conditioned fear, we then examined the effects of combined BL-BM inactivation (vehicle, 10 rats; muscimol, 10 rats). However, a possible confound here is that these rats receive twice the quantity of muscimol as in the previous experiments, potentially resulting in significant muscimol diffusion to CeM. Thus, as a control for this possibility, we added an additional group (control-muscimol; Fig. $8 C$, gray bars; see Fig. $7 C$ ) in which rats $(n=7)$ received bilateral infusions of muscimol at two sites: in BM plus a dorsal site equidistant from CeM as the $\mathrm{BL}$ infusion site (Fig. 7C). A one-way ANOVA on freezing levels during the recall test revealed a significant group effect $\left(F_{(2,24)}=\right.$ 11.45 ; $p<0.0003)$. Bonferroni-corrected post hoc $t$ tests confirmed that, 10 min after the infusion, the BM-BL muscimol group (Fig. $8 C$, black) showed much lower levels of fear expression ( $t$ test, $p=0.002$ ). Importantly, the control-muscimol group (Fig. $8 C$, gray) behaved comparably with the vehicle group during the recall test (Fig. $8 C$, white; $p=0.53$ ). However, the next day, in the same animals drug-free, both groups infused with muscimol showed a between-session extinction deficit relative to the vehicle-infused rats (one-way ANOVA, $F_{(2,24)}=4.455$, $p<0.023)$.

\section{Discussion}

This study was undertaken to test whether the basal amygdala participates in the expression of conditioned fear responses. Supporting this possibility, unit recordings revealed that a substantial proportion of BL and BM neurons acquire excitatory responses to the CS during fear conditioning. BM responses to the CS were unusual in that they persisted long after CS offset, suggesting that $\mathrm{BM}$ neurons are not passive relays of rapidly adapting LA inputs about the CS. Although separate inactivation of BL or BM shortly before testing fear recall did not reduce freezing, combined $\mathrm{BL}-\mathrm{BM}$ inactivation caused a major reduction in fear expression. Furthermore, separate or combined inactivation of BL and BM caused an extinction deficit. Together, these results suggest that the basal nuclei regulate the expression and extinction of conditioned fear responses and that there is significant functional redundancy between them.

\section{Prior studies supporting a role for the basal nuclei in conditioned fear}

There are no direct connections between LA, the input station of the amygdala for thalamic and cortical inputs about the CS (LeDoux, 2000), and CeM, the only source of amygdala projections to the periaqueductal gray, mediating freezing, and to other brainstem nuclei mediating the cardiovascular correlates of fear, such as nucleus tractus solitarius and the dorsal motor nucleus of the vagus nerve (Fig. 9) (Hopkins and Holstege, 1978; Krettek and Price, 1978; Smith and Paré, 1994; Pitkänen et al., 1997). The disconnect between LA and CeM suggests that one or more other nuclei of the amygdala are involved in relaying potentiated CS responses of LA neurons to CeM (Paré et al., 2004). The basal nuclei are good candidates to fulfill this role because they receive glutamatergic inputs from LA (Fig. 9, a) (Krettek and Price, 1978; Smith and Paré, 1994; Pitkänen et al., 1997) and send excitatory projections to CeM (Fig. 9, b) (Paré et al., 1995; Pitkänen et al., 1997). Importantly, these anatomical data were confirmed in electrophysiological studies in which BL and BM stimuli were found to elicit glutamatergic EPSPs in CeM neurons (Royer et al., 1999; Amano et al., 2010).

Although pretraining lesions of the basal nuclei were reported to have little or no effect on conditioned fear responses (Amorapanth et al., 2000; Goosens and Maren, 2001; Nader et al., 2001), posttraining lesions completely abolish them (Anglada-Figueroa and Quirk, 2005). The contrasting impact of pretraining versus posttraining lesions suggests that other structures than the basal nuclei can relay LA inputs to CeM but that, in an intact brain, the basal nuclei play a critical role in this respect. However, it was subsequently observed that inactivation of $\mathrm{BL}$ alone before testing recall does not interfere with fear expression (Herry et al., 2008).

\section{$\mathrm{CS}$ responsiveness of neurons in $\mathrm{BM}$ and $\mathrm{BL}$ during fear conditioning}

The contrasting results obtained in the latter two studies led us to hypothesize that $\mathrm{BM}$, not $\mathrm{BL}$, is the critical relay station between LA and CeM. In keeping with this, unit recordings revealed that one-third of BM cells acquired robust excitatory responses to the $\mathrm{CS}$ as a result of fear conditioning. Moreover, the next day during the recall test, the same proportion of BM neurons displayed excitatory CS responses. As previously observed in the dorsal part of LA (Quirk et al., 1995), the CS responses of most BM neurons vanished during the extinction training session, paralleling the rapid within-session reduction in freezing. At odds with dorsal LA neurons, however, the CS responses of BM neurons often outlasted the CS by up to $80 \mathrm{~s}$ during the recall test, suggesting that BM neurons are not mere relays of potentiated LA inputs to CeM but that through interactions with each other or with one of more other structures, such as the prelimibic cortex (Fig. 9, c and d) (Burgos-Robles et al., 2009), they actively modify and extend the CS-related signals they receive from LA.

Since BM neurons project to CeM, these findings are consistent with the notion that they contribute to the excitation of CeM neurons by the CS (Ciocchi et al., 2010; Duvarci et al., 2011). However, at odds with the idea that $\mathrm{BM}$, not $\mathrm{BL}$, plays a role in conditioned fear expression, a similar proportion of CS-responsive cells was found in BL, as previously reported (Herry et al., 2008). As a result, it remained unclear why combined BL-BM lesions (Anglada-Figueroa and Quirk, 2005) versus inactivation of BL only (Herry et al., 2008) have such different outcomes.

\section{Functionally redundant contributions of $B M$ and $B L$ to the expression of conditioned fear}

To shed light on this question, we compared the effects of separate versus combined inactivation of BL and BM with local muscimol infusions just before testing fear recall. While separate inactivation of $\mathrm{BL}$ or $\mathrm{BM}$ did not reduce conditioned freezing, combined inactivation of the basal nuclei did. This suggests that 

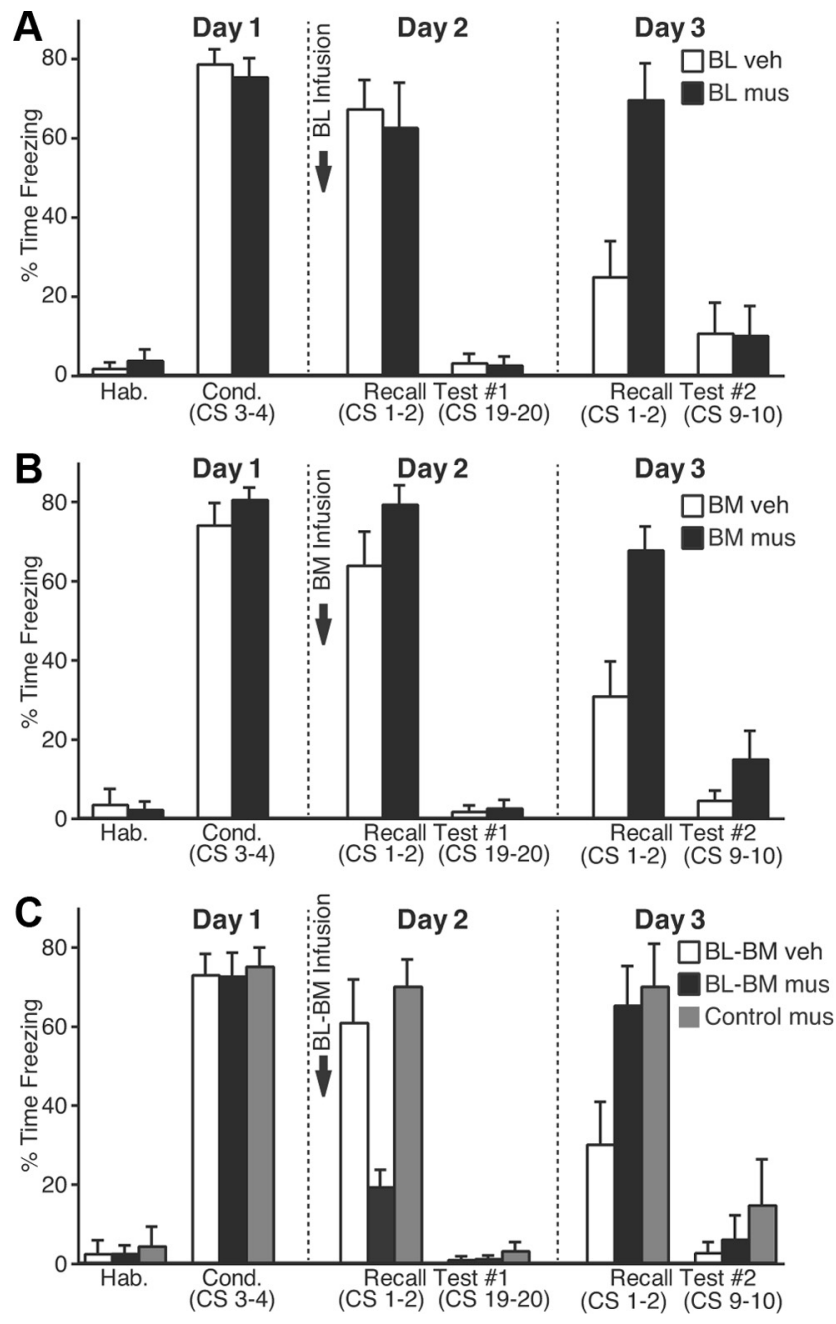

Figure 8. Impact of muscimol infusions in the basal nuclei on fear expression. $A-C$, CS-elicited freezing ( $y$-axis; average \pm SEM) during the behavioral protocol ( $x$-axis). Ten minutes before testing recall, we performed bilateral infusions of vehicle (empty bars) or muscimol (filled bars) in $B L(A), B M$ (B), or both (O). C, Gray bars, Rats that received muscimol in BM plus a site dorsal to Ce (Fig. 7C).

there is functional redundancy between BM and BL such that inactivating only one of the two has no effect on conditioned fear. Since freezing is an all-or-none phenomenon, there is likely a threshold function in the amount of CeM excitation required to elicit this conditioned response. Below this threshold, as when BL and BM are simultaneously inactivated, the CS would not evoke freezing. Above this threshold, as in normal conditions or when $\mathrm{BL}$ and $\mathrm{BM}$ are separately inactivated, conditioned freezing would be observed because BL and BM independently produce sufficient depolarization of CeM cells to trigger freezing. However, it should be noted that, while the above might apply to the simplified paradigm studied here, in more complex conditions, BL and BM, via their contrasting connections, might endow the fear system with additional flexibility, rather than being simply redundant. On the input side, for instance, infralimbic and prelimbic axons show minimal overlap in BL and BM (McDonald, 1998), raising the possibility that they are differentially regulated in some conditions.

\section{Contributions of the basal nuclei to the extinction of conditioned fear responses}

Previous studies reported that inactivation of either BL alone (Herry et al., 2008) or LA and BL (Sierra-Mercado et al., 2011) interfered with extinction. However, in both cases, muscimol also

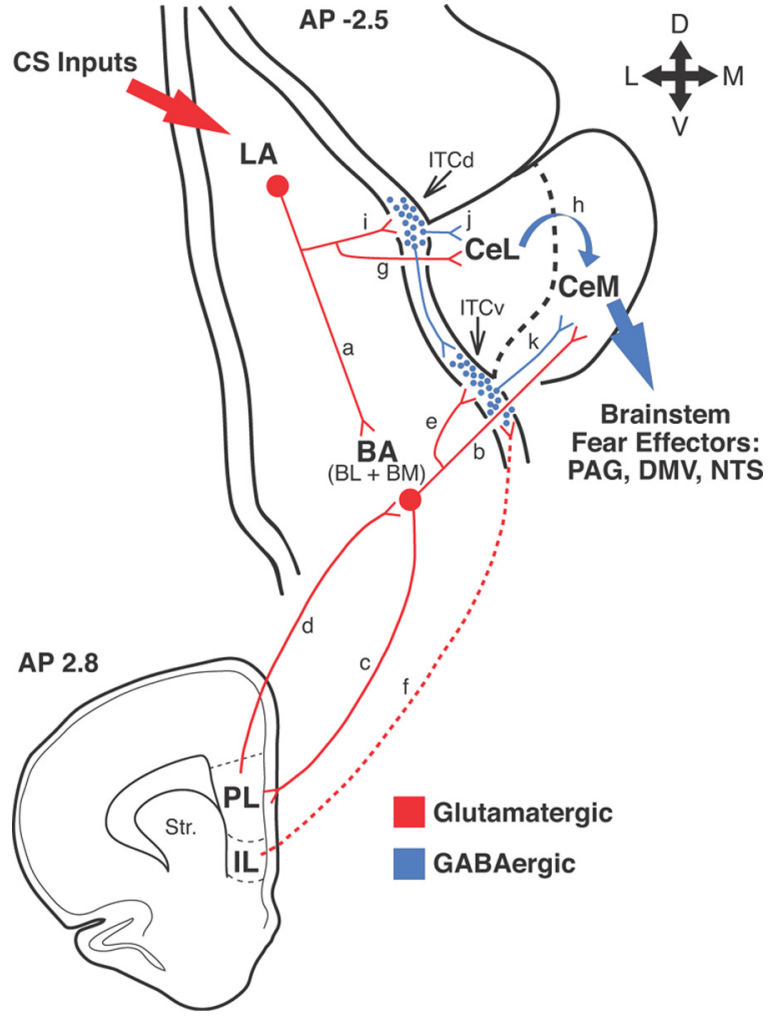

Figure 9. Network interactions supporting the acquisition and extinction of conditioned fear responses. Thalamic and cortical afferents convey CS information to LA (large red arrow on top left). CeM constitutes the main source of amygdala projections to brainstem fear effectors (large blue arrow on right). However, LA has no direct connections to CeM neurons. LA can influence CeM indirectly via the basal nuclei $(B A)(a, b)$ or central lateral $(C e L)$ nucleus $(g, h)$. Our results support the notion that in an intact brain, LA can excite CeM neurons via the basal nuclei. However, if the BA nuclei are lesioned before training, conditioning occurs normally, suggesting that CeL might also participate. Disinhibition of CeM from inputs arising in CeL might be involved (Ciocchi et al., 2010; Duvarci et al., 2011). Interactions between LA, BA, and Ce are regulated by intercalated (ITC) cells located at the BLA-Ce border (blue circles) as well as the infralimbic (IL) and prelimbic (PL) regions of the medial prefrontal cortex (bottom scheme). In rats, the medial ITC cells occur in multiple main clusters: one located lateral to CeL (ITCd) and others located more ventrally (ITCv). ITCd receives glutamatergic inputs from LA (i) and project to CeL (j). ITCv receives glutamatergic inputs from BA (e) and project to $C \mathrm{CM}(\mathrm{k})$. In addition, ITCd projects to ITCv. After fear conditioning, via LA neurons, the CS excites ITCd neurons more strongly, likely resulting in the inhibition of CeL cells and disinhibition of CeM neurons. The increased recruitment of ITCd cells would also cause the inhibition of ITCv neurons, resulting in further CeM disinhibition. These two disinhibitory mechanisms would reinforce the glutamatergic excitation of CeM by BA inputs. It is likely that the persistence of BM responses after CS offset depends on interactions with the prelimbic cortex ( $c, d)$. In contrast, IL would regulate extinction by enabling a potentiation of BA inputs to ITC neurons ( $f$ ), resulting in increased feedforward inhibition of CeM cells by ITC cells (e, k). PAG, Periaqueductal gray; DMV, dorsal motor nucleus of the vagus; NTS, nucleus of the solitary tract.

altered fear expression during extinction training (albeit in opposite directions), complicating the interpretation of these results. In contrast, we observed that, with the low muscimol quantity used here, inactivation of BL or BM just before fear recall caused a between-session extinction deficit despite no change in fear expression during extinction training. This suggests that inactivation of basal amygdala neurons interfered with extinctionrelated plasticity, either locally and/or in one or more of their target(s). With respect to the latter possibility, likely candidates include infralimbic (Quirk and Mueller, 2008) and intercalated neurons. In support of this, it was reported that extinction training causes a potentiation of BL inputs to intercalated cells (Fig. 9, e), an effect that required infralimbic activity (Fig. 9, f) during 
and/or shortly after extinction training and caused increased feedforward inhibition in CeM (Amano et al., 2010).

Only one study (Herry et al., 2008) reported on the behavior of basal amygdala neurons during extinction and only in BL, not BM, neurons. In this study, three types of BL neurons were distinguished. A first cell type, termed fear neurons (17\% of cells), acquired responses to the CS as a result of fear conditioning and lost them following extinction training. A second class, termed extinction neurons $(14 \%)$, remained unresponsive to the CS after conditioning but acquired CS responses following extinction training. Finally, a third class, termed extinction-resistant neurons (26\%), acquired responses to the CS as a result of fear conditioning but displayed persistent CS responses throughout extinction.

Our results partially overlap with these earlier findings. In BL and $\mathrm{BM}$, fear neurons were prevalent, followed by extinctionresistant neurons ( $\sim 25$ and $10 \%$ of cells, respectively). Although we observed very few extinction cells (3\%), this may be attributable to differences in the paradigm and/or species used. Indeed, the study by Herry et al. (2008) was performed in mice, involved a more intense extinction training paradigm, and used repeated pips as a CS rather than a prolonged tone. Regarding the last factor, it is conceivable that extinction cells have rapidly adapting auditory responses that are missed or underestimated with prolonged tones.

In any event, the rapid extinction-related shifts in the CS responsiveness of many BL and BM fear neurons, from positive responses to none or even inhibition, coupled to the presence of a few extinction cells suggest that fear extinction depends not only on the infralimbic-dependent feedforward inhibition of CeM neurons by intercalated cells but also on rapid shifts in synaptic weights locally in the basal nuclei. The increased inhibition of basal amygdala neurons that develops within a single extinction training session points to an important role of the local GABAergic circuitry, consistent with earlier studies that revealed increased expression of several GABAergic markers including GABA-synthesizing enzyme, $\mathrm{GABA}_{\mathrm{A}}$ receptor subunits and $\mathrm{a} \mathrm{GABA}_{\mathrm{A}}$ receptor clustering protein (Chhatwal et al., 2005; Heldt and Ressler, 2007).

Challenges for future studies include analyzing extinctionrelated $\mathrm{BL}$ and $\mathrm{BM}$ responsiveness to conditioned pips in rats and identifying the network interactions allowing a subset of basal amygdala neurons to transform the transient CS signal they receive from LA into sustained CS responses.

\section{References}

Amano T, Unal CT, Paré D (2010) Synaptic correlates of fear extinction in the amygdala. Nat Neurosci 13:489-494.

Amorapanth P, LeDoux JE, Nader K (2000) Different lateral amygdala outputs mediate reactions and actions elicited by a fear-arousing stimulus. Nat Neurosci 3:74-79.

Anglada-Figueroa D, Quirk GJ (2005) Lesions of the basal amygdala block expression of conditioned fear but not extinction. J Neurosci 25:9680-9685.

Burgos-Robles A, Vidal-Gonzalez I, Quirk GJ (2009) Sustained conditioned responses in prelimbic prefrontal neurons are correlated with fear expression and extinction failure. J Neurosci 29:8474-8482.

Cassell MD, Gray TS, Kiss JZ (1986) Neuronal architecture in the rat central nucleus of the amygdala: a cytological, hodological, and immunocytochemical study. J Comp Neurol 246:478-499.

Chhatwal JP, Myers KM, Ressler KJ, Davis M (2005) Regulation of gephyrin and $\mathrm{GABA}_{\mathrm{A}}$ receptor binding within the amygdala after fear acquisition and extinction. J Neurosci 25:502-506.

Ciocchi S, Herry C, Grenier F, Wolff SB, Letzkus JJ, Vlachos I, Ehrlich I, Sprengel R, Deisseroth K, Stadler MB, Müller C, Lüthi A (2010) Encoding of conditioned fear in central amygdala circuits. Nature 468:277-282.

Davis M (1992) The role of the amygdala in fear and anxiety. Annu Rev Neurosci 15:353-375.

Duvarci S, Popa D, Paré D (2011) Central amygdala activity during fear conditioning. J Neurosci 31:289-294.
Goosens KA, Maren S (2001) Contextual and auditory fear conditioning are mediated by the lateral, basal, and central amygdaloid nuclei in rats. Learn Mem 8:148-155.

Gray TS, Magnuson DJ (1987) Neuropeptide neuronal afferents from the bed nucleus of the stria terminalis and central amygdaloid nucleus to the dorsal vagal complex in the rat. J Comp Neurol 262:365-374.

Heldt SA, Ressler KJ (2007) Training-induced changes in the expression of $\mathrm{GABA}_{\mathrm{A}}$-associated genes in the amygdala after the acquisition and expression of pavlovian fear. Eur J Neurosci 26:3631-3644.

Herry C, Ciocchi S, Senn V, Demmou L, Müller C, Lüthi A (2008) Switching on and off fear by distinct neuronal circuits. Nature 454:600-606.

Hopkins DA, Holstege G (1978) Amygdaloid projections to the mesencephalon, pons and medulla oblongata in the cat. Exp Brain Res 32:529-547.

Krettek JE, Price JL (1978) A description of the amygdaloid complex in the rat and cat with observations on intra-amygdaloid axonal connections. J Comp Neurol 178:255-280.

LeDoux JE (2000) Emotion circuits in the brain. Annu Rev Neurosci 23:155-184

LeDoux JE, Cicchetti P, Xagoraris A, Romanski LM (1990) The lateral amygdaloid nucleus: sensory interface of the amygdala in fear conditioning. J Neurosci 10:1062-1069.

McDonald AJ (1998) Cortical pathways to the mammalian amygdala. Prog Neurobiol 55:257-332.

Nader K, Majidishad P, Amorapanth P, LeDoux JE (2001) Damage to the lateral and central, but not other, amygdaloid nuclei prevents the acquisition of auditory fear conditioning. Learn Mem 8:156-163.

Pape HC, Paré D (2010) Plastic synaptic networks of the amygdala for the acquisition, expression, and extinction of conditioned fear. Physiol Rev 90:419-463.

Paré D, Smith Y, Paré JF (1995) Intra-amygdaloid projections of the basolateral and basomedial nuclei in the cat: Phaseolus vulgaris-leucoagglutinin anterograde tracing at the light and electron microscopic level. Neuroscience 69:567-583.

Paré D, Quirk GJ, LeDoux JE (2004) New vistas on amygdala networks in conditioned fear. J Neurophysiol 92:1-9.

Pitkänen A, Savander V, LeDoux JE (1997) Organization of intraamygdaloid circuitries in the rat: an emerging framework for understanding functions of the amygdala. Trends Neurosci 20:517-523.

Phelps EA, LeDoux JE (2005) Contributions of the amygdala to emotion processing: from animal models to human behavior. Neuron 48:175-187.

Quirk GJ, Mueller D (2008) Neural mechanisms of extinction learning and retrieval. Neuropsychopharmacology 33:56-72.

Quirk GJ, Repa C, LeDoux JE (1995) Fear conditioning enhances shortlatency auditory responses of lateral amygdala neurons: parallel recordings in the freely behaving rat. Neuron 15:1029-1039.

Rizvi TA, Ennis M, Behbehani MM, Shipley MT (1991) Connections between the central nucleus of the amygdala and the midbrain periaqueductal gray: topography and reciprocity. J Comp Neurol 303:121-131.

Romanski LM, Clugnet MC, Bordi F, LeDoux JE (1993) Somatosensory and auditory convergence in the lateral nucleus of the amygdala. Behav Neurosci 107:444-450.

Royer S, Martina M, Paré D (1999) An inhibitory interface gates impulse traffic between the input and output stations of the amygdala. J Neurosci 19:10575-10583.

Sierra-Mercado D, Padilla-Coreano N, Quirk GJ (2011) Dissociable roles of prelimbic and infralimbic cortices, ventral hippocampus, and basolateral amygdala in the expression and extinction of conditioned fear. Neuropsychopharmacology 36:529-538.

Smith Y, Paré D (1994) Intra-amygdaloid projections of the lateral nucleus in the cat: PHA-L anterograde labeling combined with post-embedding GABA and glutamate immunocytochemistry. J Comp Neurol 342:232-248.

Turner BH, Herkenham M (1991) Thalamoamygdaloid projections in the rat: a test of the amygdala's role in sensory processing. J Comp Neurol 313:295-325.

Veening JG, Swanson LW, Sawchenko PE (1984) The organization of projections from the central nucleus of the amygdala to brainstem sites involved in central autonomic regulation: a combined retrograde transport-immunohistochemical study. Brain Res 303:337-357.

Wilensky AE, Schafe GE, Kristensen MP, LeDoux JE (2006) Rethinking the fear circuit: the central nucleus of the amygdala is required for the acquisition, consolidation, and expression of pavlovian fear conditioning. J Neurosci 26:12387-12396. 